\title{
CENTERS OF DISKS IN RIEMANNIAN MANIFOLDS
}

\author{
IGOR BELEGRADEK AND MOHAMMAD GHOMI
}

\begin{abstract}
We prove the existence of a center, or continuous selection of a point, in the relative interior of $C^{1}$ embedded $k$-disks in Riemannian $n$-manifolds. If $k \leq 3$ the center can be made equivariant with respect to the isometries of the manifold, and under mild assumptions the same holds for $k=4=n$. By contrast, for every $n \geq k \geq 6$ there are examples where an equivariant center does not exist. The center can be chosen to agree with any of the classical centers defined on the set of convex compacta in the Euclidean space.
\end{abstract}

\section{INTRODUCTION}

There are several distinguished points inside a compact convex subset of the Euclidean space, see [KMT91], [Mos06, Chapter 12], and [Sch14, Section 5.4.1]. In this paper we investigate if there is a geometrically meaningful point inside every embedded disk in a Riemannian manifold. Any such point should depend continuously on the disk and be equivariant under isometries.

To set the stage let $G$ be a subgroup of the isometry group of a smooth (i.e., $C^{\infty}$ ) connected Riemannian manifold $M$, and $\mathcal{X}(M)$ be the space of compact connected $C^{1}$ embedded submanifolds of $M$ equipped with the $C^{1}$ topology (see Section 3). For a subspace $X$ of $\mathcal{X}(M)$, a center is a continuous map $\mathfrak{c}: X \rightarrow M$ such that $\mathfrak{c}(D)$ lies in the relative interior of $D$ for every $D \in X$. If in addition $X$ is $G$-invariant and $\mathfrak{c}$ is $G$-equivariant, we say that $\mathfrak{c}$ is $G$-equivariant, which simply means that $\mathfrak{c}(g D)=g \mathfrak{c}(D)$ for all $D \in X, g \in G$. We investigate the existence of $\mathfrak{c}$ for a given triple $(M, G, X)$.

The best-known examples of a center are the center of mass and the Steiner point, which we call the classical centers. They are Iso $\left(\mathbb{R}^{n}\right)$-equivariant centers on the space of convex compacta in $\mathcal{X}\left(\mathbb{R}^{n}\right)$, where $\operatorname{Iso}\left(\mathbb{R}^{n}\right)$ is the group of Euclidean isometries, see [Sch14].

To state our findings, for any $D \in \mathcal{X}(M)$ let $G_{D}$ be the subgroup of $G$ which maps $D$ to itself, and $D^{G_{D}}$ be the points of $D$ which are fixed by $G_{D}$ (see Section 2). Note

Date: July 16, 2019 (Last Typeset).

2010 Mathematics Subject Classification. Primary: 53C40, 54C65; Secondary: 57S25, 52A20.

Key words and phrases. Continuous selection, equivariant, proper actions, actions on disks.

The research of M. G. was supported in part by NSF grants DMS-1308777 and DMS-1711400. 
that if $\mathfrak{c}$ is $G$-equivariant, then $\mathfrak{c}(D) \in D^{G_{D}}$, and in particular, no $G$-equivariant center exists if $D^{G_{D}}=\emptyset$ for some $D \in X$. Our main result is as follows:

Theorem 1.1. The space of submanifolds $D \in \mathcal{X}(M)$ such that $D^{G_{D}}$ is contractible admits a $G$-equivariant center. Furthermore, this center can be chosen to agree with any given $G$-equivariant center defined on a closed subset $A$ of $\mathcal{X}(M)$.

An important example of $A$ is the set of convex compacta in $\mathbb{R}^{n}$ equipped with one of the classical centers mentioned above. In this case our results are summarized in Corollary 1.7 below.

We prove Theorem 1.1 by reducing the problem to finding sections of certain bundles with $D^{G_{D}}$ as fibers, and then fitting the sections together to define a global center. The construction involves some choices, and the resulting center is not canonical.

Let us review some natural conditions under which $D^{G_{D}}$ is contractible, which yield the following corollaries of Theorem 1.1. It is to be understood that each of these corollaries has a relative version, i.e., the corresponding center can be chosen to agree with any given $G$-equivariant center defined on a closed subset of $\mathcal{X}(M)$. First note that if $G$ is trivial, then $D^{G_{D}}=D$. So we obtain:

Corollary 1.2. The space of contractible submanifolds $D \in \mathcal{X}(M)$ admits a center.

Another condition that ensures contractibility of $D^{G_{D}}$ is that each $D \in \mathcal{X}(M)$ is homeomorphic to a disk of dimension $\leq 3$. This is due to the fact that any smooth action of a compact Lie group on a disk of dimension $\leq 3$ is smoothly equivalent to a linear action, which implies that $D^{G_{D}}$ is a disk. For actions on 2-disks linearity is a standard consequence of the uniformization theorem (see the beginning of Appendix A), while the 3-dimensional case is established in [KS92, Theorem B].

Let $\mathfrak{D}^{k}(M)$ be the subspace of $D \in \mathcal{X}(M)$ such that $D$ is homeomorphic to a disk of dimension $\leq k$. From the previous paragraph we obtain:

Corollary 1.3. $\mathfrak{D}^{3}(M)$ admits a $G$-equivariant center.

On the other hand, contractibility of $D^{G_{D}}$ generally fails when $D$ is homeomorphic to a 4 -disk, because then $D^{G_{D}}$ may be an acyclic non-contractible 3 -manifold, see Lemma A.4. For this case to occur $G_{D}$ must be generated by an involution whose action on $D$ reverses the orientation, see Lemmas A.1 and A.3. Avoiding this case, we obtain the existence of centers in dimension 4 :

Corollary 1.4. If $\operatorname{dim}(M)=4$ and the $G$-action on $M$ is orientation-preserving, then $\mathfrak{D}^{4}(M)$ admits a $G$-equivariant center.

Another result for 4-manifolds can be obtained by observing that no compact acyclic non-contractible 3-manifold smoothly embeds into $S^{3}$ due to the topological Schoenflies theorem [Bro60]. In Lemma 4.2 we note that $D^{G_{D}}$ is a $C^{1}$ submanifold 
of $M^{G_{D}}$, the fixed point set of $G_{D}$ in $M$. Therefore, a non-contractible $D^{G_{D}}$ cannot occur if for any involution in $G$ its fixed point set in $M$ smoothly embeds into $S^{3}$. The latter can be forced by the following geometric assumptions.

Corollary 1.5. If either $M=S^{4}$ with its standard action of $G=O(5)$, or $M$ is a Hadamard 4-manifold with isometry group $G$, then $\mathfrak{D}^{4}(M)$ admits a $G$-equivariant center.

Recall that a Hadamard manifold is a contractible complete Riemannian manifold of nonpositive sectional curvature, and the fixed point set of any isometric action on a Hadamard manifold is diffeomorphic to a Euclidean space, due to the CartanHadamard theorem.

The above 4-dimensional results depend on establishing that the fixed point set of a smooth compact Lie group action on the 4-disk is either a disk or a compact acyclic 3-manifold. We check this in Lemmas A.1 and A.3. A key ingredient is that any smooth finite group action on the 4-disk fixes a point. This was established in [BKS90, Theorem II.1] modulo an announcement of Thurston that any nonfree smooth finite group action on $S^{3}$ preserves a round metric. Thurston's claim was finally proved in [DL09] via a Ricci flow arguments, and his outline was made rigorous in [BLP05, Corollary 1.1] for orientation-preserving actions.

In dimension 5 the situation is unclear, e.g., it does not seem to be known whether every smooth action of a compact Lie group on a 5-disk has a fixed point. The strategy used in Corollary 1.5 for linear actions on $\mathbb{R}^{4}$ breaks down one dimension up, because there is a linear involution of $\mathbb{R}^{5}$ whose fixed point set on some embedded disk is not contractible, see Lemma A.5.

Starting from dimension 6 an equivariant center need not exist, namely, there are triples $(M, X, G)$ such that $D^{G_{D}}=\emptyset$ for some smoothly embedded disk $D \in X$, which rules out the existence of a $G$-equivariant center, as we had mentioned earlier. Indeed, for each $n \geq 6$ there is a smooth action of the alternating group $A_{5}$ on $S^{n}$ with exactly one fixed point, see [BM05] and references therein, and excising an invariant tubular neighborhood of this point yields a fixed point free smooth action of $A_{5}$ on the $n$-disk. One can then embed the resulting $A_{5}$-action on the $n$-disk into a fixed point free $A_{5}$-action on a boundaryless manifold, e.g., into $\mathbb{R}^{n}$ by attaching a collar along the boundary, or into $S^{n}$ by doubling along the boundary.

The Mostow-Palais theorem [Bre72, Theorem VI.4.1] yields a smooth embedding of the above fixed point free $A_{5}$-action on the 6 -disk into an orthogonal $A_{5}$-action on some $\mathbb{R}^{r}$. (With some effort one can obtain an explicit upper bound for $r$ but we will not attempt it here because we are unable to determine the optimal $r$.) As we shall explain at the end of Section 4, this discussion easily implies the following:

Proposition 1.6. For any $m, n$ such that either $m \geq n+r-6 \geq r$ or $m \geq n \geq r$ there is an orthogonal $A_{5}$-action on $\mathbb{R}^{m}$ that preserves a smoothly embedded $n$-disk on which $A_{5}$ acts without a fixed point. In particular, $\mathfrak{D}^{n}\left(\mathbb{R}^{m}\right) \backslash \mathfrak{D}^{n-1}\left(\mathbb{R}^{m}\right)$ does not admit an $O(m)$-equivariant center. 
At this point it seems worthwhile to summarize our results for the Euclidean space:

Corollary 1.7. Any $\operatorname{Iso}\left(\mathbb{R}^{n}\right)$-equivariant center on the set of convex compacta in $\mathbb{R}^{n}$ extends to a center $\mathfrak{c}: \mathfrak{D}^{n}\left(\mathbb{R}^{n}\right) \rightarrow \mathbb{R}^{n}$ that is $\operatorname{Iso}\left(\mathbb{R}^{n}\right)$-equivariant on $\mathfrak{D}^{3}\left(\mathbb{R}^{n}\right)$. Moreover, if $n=4$, then $\mathfrak{c}$ is $\operatorname{Iso}\left(\mathbb{R}^{4}\right)$-equivariant on $\mathfrak{D}^{4}\left(\mathbb{R}^{4}\right)$. If $k$ is sufficiently large, then $\mathfrak{D}^{k}\left(\mathbb{R}^{n}\right)$ does not admit an $\operatorname{Iso}\left(\mathbb{R}^{n}\right)$-equivariant center.

The results of this paper belong to the subject of continuous selections of multivalued mappings, an established branch of topology which was pioneered by Michael [Mic56a, Mic56b, Mic59] and extensively surveyed in [RS98, RS02, RS14]. Indeed, assigning to each $D \in \mathcal{X}(M)$ the relative interior of $D^{G_{D}}$ in $M$ yields a multivalued map $\mathcal{X}(M) \rightarrow M$ which is lower semicontinuous in the sense of [RS98, Definition 0.43]. What we call a $G$-equivariant center is a continuous $G$-equivariant selection of this multivalued map over a subset $X$ of $\mathcal{X}(M)$. The theory of continuous selections implies existence of a non-equivariant center under certain assumptions on $X \subset \mathcal{X}(M)$ that tend to come in two flavors: either every $D \in X$ needs to satisfy a suitable generalized convexity condition, or $X$ is required to be finite-dimensional (see [RS98] for details). By contrast, our focus is on equivariant centers, and there seems to be no prior work analogous to the results of this paper.

The classical centers are continuous in the Hausdorff topology on the set of convex compacta, and hence one might expect that this would be a natural topology for $\mathcal{X}(M)$. However, in Remark 3.4 we shall see that Theorem 1.1 fails when $\mathcal{X}\left(\mathbb{R}^{n}\right)$ is given the Hausdorff topology. On the other hand, for any $k$ the Hausdorff topology on the set of $k$-dimensional convex compacta in $\mathcal{X}\left(\mathbb{R}^{n}\right)$ coincides with the $C^{0}$ topology, and hence we ask:

Question 1.8. Does Theorem 1.1 remain valid when $\mathcal{X}(M)$ is replaced with the space of $C^{0}$ submanifolds equipped with the $C^{0}$ topology?

\section{BACKGROUND ON GROUP ACTIONS}

Here we review the basic facts on Lie group actions used in this work. Throughout this section $G$ is a Lie group and $X$ is a metrizable space. A $G$-action on $X$ is a continuous map $a: G \times X \rightarrow G$, written as $g x:=a(g, x)$, such that the map $g \rightarrow a(g, \cdot)$ is a homomorphism between $G$ and the homeomorphism group of $X$. A space with a $G$-action is a $G$-space. The orbit space $X / G$ is the set of $G$-orbits with the quotient topology. A map $f: X \rightarrow Y$ of $G$-spaces is a $G$-map if $f$ is continuous and $f(g x)=g f(x)$ for all $x \in X$, and $g \in G$; we will also refer to $f$ as $G$-equivariant or just equivariant when $G$ is understood. For $S \subseteq X$, we let $g S:=\{g x: x \in S\}$ and use the following notations:

$$
\begin{gathered}
G_{S}:=\{g \in G: g S=S\}=\text { the isotropy subgroup of } S \text { in } G, \\
G S:=\{g S: g \in G\}=\text { the } G \text {-orbit of } S, \\
X^{G}:=\{x \in X: g x=x \text { for each } g \in G\}=\text { the fixed point set of } G \text { in } X .
\end{gathered}
$$


If $S=\{x\}$, then $G S, G_{S}$ are denoted by $G x, G_{x}$, respectively. Note that $G_{x}$ is a closed subgroup of $G$. If $G S=S$, then $S$ is $G$-invariant. If $x \in S_{x} \subseteq X$, then $S_{x}$ is called a $G_{x}$-slice at $x$ provided that $G S_{x}$ is open in $X$ and there is a $G$-map $f: G S_{x} \rightarrow G / G_{x}$ with $S_{x}=f^{-1}\left(G_{x}\right)$.

Lemma 2.1. Let $\alpha: A \rightarrow X$ be a $G$-map of $G$-spaces with $\alpha(a)=x$ and $G_{a}=G_{x}$. If $S_{x}$ is a $G_{x}$-slice at $x$, then $\alpha^{-1}\left(S_{x}\right)$ is a $G_{a}$-slice at $a$.

Proof. Note that $\alpha \circ f: A \rightarrow G / G_{x}=G / G_{a}$ is a $G$-map with $(\alpha \circ f)^{-1}\left(G_{a}\right)=$ $\alpha^{-1}\left(S_{x}\right)$. For any $g \in G, z \in X$, we have $g \alpha^{-1}(z)=\alpha^{-1}(g z)$, and hence $G \alpha^{-1}\left(S_{x}\right)=\alpha^{-1}\left(G S_{x}\right)$. Thus openness of $G S_{x}$ implies openness of $G \alpha^{-1}\left(S_{x}\right)$.

A $G$-space $X$ is Palais-proper if any $x \in X$ has a neighborhood $V_{x}$ such that every $y \in Y$ has a neighborhood $V_{y}$ for which $\left\{g \in G: g V_{x} \cap V_{y} \neq \emptyset\right\}$ is precompact in $G$. For example, if $G$ is compact, then any $G$-space is Palais-proper. If $G$ is an isometry group of a smooth Riemannian manifold, then the $G$-space is Palais-proper [KN96, Theorem I.4.7], and conversely, any smooth Palais-proper action preserves a smooth Riemannian metric [Pal61, Theorem 4.3.1].

Note that if $x$ is a point of a Palais-proper $G$-space $X$, then $G_{x}$ is compact (because it is closed and precompact in $G$ ). A key result established in [Pal61, Section 2.3] is that every point $x$ in a Palais-proper $G$-space is contained in a $G_{x}$-slice. In [Pal61, Section 2.1] one finds the following characterization of slices at points with compact isotropy subgroups:

Lemma 2.2 ([Pal61]). Let $x \in S \subseteq X$ and suppose that $G_{x}$ is compact. Then $S$ is a $G_{x}$-slice at $x$ if and only if the following conditions hold:

(a) $g S \cap S \neq \emptyset$ if and only if $g \in G_{x}$,

(b) $S$ is closed in $G S$, and $G S$ is open in $X$,

(c) there is an open set $O$ such that $S \subset O \subset G S$ and $\{g \in G: g O \cap O \neq \emptyset\}$ is precompact in $G$.

The following lemma summarizes what we need to know about slices.

Lemma 2.3. If $S_{x}$ is a $G_{x}$-slice at $x$ and $G_{x}$ is compact, then the following statements hold:

(i) $G_{y} \subset G_{x}$ for every $y \in S_{x}$.

(ii) If $W$ is open in a slice $S_{x}$, then $G W$ is open in $X$.

(iii) The inclusion induced map $S_{x} / G_{x} \rightarrow X / G$ is an open embedding.

(iv) Any open $G_{x}$-invariant neighborhood of $x$ in $S_{x}$ is a $G_{x}$-slice at $x$.

(v) Any neighborhood of $x$ in $S_{x}$ contains an open set that is a $G_{x}$-slice at $x$. 
(vi) For any neighborhood $U$ of the identity in $G$ there is a neighborhood $V$ of $x$ in $G S$ such that for each $y \in V$ there is $u \in U$ with $u^{-1} G_{y} u \subseteq G_{x}$.

Proof. (i) is immediate by Lemma 2.2(a), while (ii) is proved in [Pal61, Corollary on p.306]. The rest of the items are established as follows:

(iii): The map here is one-to-one by Lemma 2.2(a). Further, it is a homeomorphism by (ii) and the defining properties of the quotient topology.

(iv): Let $W$ be an open $G_{x}$-invariant neighborhood of $x$ in $S_{x}$, and appeal to Lemma 2.2. The conditions (a) and (c) are immediate, and (i) implies that $G W$ is open in $X$. To see that $W$ is closed in $G W$ take $w_{i} \rightarrow g w$, where $g \in G$, $w_{i}, w \in W$, and note that $w, w_{i} \in S_{x}$ implies $g w \in S_{x}$, so $g \in G_{x}$ and hence $g w \in W$.

(v): Note that $S_{x}$ has a $G_{x}$-invariant metric [Pal60, Proposition 1.1.12], so any open metric ball centered at $x$ is $G_{x}$-invariant, and hence is a slice by (iii). Any neighborhood of $x$ contains such a ball.

(vi): Choose $V$ inside $O$ of Lemma 2.2(c). Then $G_{x}, G_{y}$ lie in a compact subgroup of $G$, in which case a proof can be found in [Bre72, Corollary II.5.5].

\section{Space of Submanifolds}

In this section we give a precise definition of the topology on $\mathcal{X}(M)$. Further we show that this topology is Palais-proper and induced by a $G$-invariant metric. For any submanifold $D \in \mathcal{X}(M)$ let $\mathcal{X}_{D}(M) \subset \mathcal{X}(M)$ be the collection of submanifolds which are $C^{1}$ diffeomorphic to $D$. In other words,

$$
\mathcal{X}_{D}(M):=\operatorname{Emb}^{1}(D, M) / \operatorname{Diff}^{1}(D),
$$

the space of $C^{1}$ embeddings of $D$ into $M$ modulo $C^{1}$ diffeomorphisms of $D$. We equip $\mathcal{X}_{D}(M)$ with its standard $C^{1}$ topology, which is induced by $C^{1}(D, M)$, the space of $C^{1}$ mappings $D \rightarrow M$. Thus a pair of submanifolds $A, B \in \mathcal{X}_{D}(M)$ are close if they admit parametrizations $f, g \in \operatorname{Emb}^{1}(D, M)$ that are $C^{1}$ close. Finally we topologize $\mathcal{X}(M)$ as the disjoint union of $\mathcal{X}_{D}(M)$ where $D$ ranges over $C^{1}$ diffeomorphism classes of submanifolds $D \in \mathcal{X}(M)$. Note that the obvious $G$-action on $\mathcal{X}(M)$ given by $\alpha(g, D)=g D$ is effective.

Lemma 3.1. The following statements hold:

(1) The topology on $\mathcal{X}_{D}(M)$ is induced by a $G$-invariant metric $d_{D}$.

(2) The $G$-space $\mathcal{X}_{D}(M)$ is Palais-proper.

(3) The orbit space $\mathcal{X}_{D}(M) / G$ is metrizable.

Proof. A convenient way to describe $\mathcal{X}_{D}(M)$ is to consider a smooth $G$-equivariant embedding of $M$ into a Hilbert space $\mathcal{H}$ equipped with some orthogonal $G$-action, 
see [Kan94, Theorem 0.1]. In general, one cannot equivariantly embed $M$ into a finite dimensional linear space; this can be done, e.g., if both $G$ and $M$ are compact [Bre72, Theorem VI.4.1].

To prove (1) we define a $G$-invariant metric $d_{D}$ on $\mathcal{X}_{D}(\mathcal{H})$, and then restrict it to a smoothly embedded $G$-invariant copy of $M$ in $\mathcal{H}$. Define $d_{D}(A, B)$ as the infimum of $\|\alpha-\beta\|_{C^{1}}$ taken over all $C^{1}$ embeddings $\alpha, \beta \in C^{1}(D, \mathcal{H})$ with images $A, B$, respectively. Here the $C^{1}$ norm is computed using the norm on $\mathcal{H}$. The triangle inequality follows from the one for $\mathcal{H}$ and properties of the infimum, and the $G$-invariance holds because the $G$-action on $\mathcal{H}$ preserves the norm. For nondegeneracy note that if say $a \in A \backslash B$, then $d_{D}(A, B)$ is bounded below by the distance from $a$ to $B$, hence $d_{D}(A, B)=0$ implies $A=B$.

To prove (2) fix $r \in(0,1)$ and let $V_{x}$ be the $r$-ball about $x$ in $\left(\mathcal{X}_{D}(M), d\right)$. If $A, B \in \mathcal{X}_{D}(M)$ with $V_{A} \cap g V_{B} \neq \emptyset$, then $g B$ lies in the $3 r$-neighborhood of $A$, and since the $G$-action on $M$ is Palais-proper, so is the $G$-action on $\mathcal{X}(M)$.

(3) is proved in [Pal61, Theorem 4.3.4] assuming (1)-(2) with the metric given by $\bar{d}_{D}(G x, G y)=\inf \left\{d_{D}(x, g y): g \in G\right\}$.

Remark 3.2. The proof of Lemma 3.1 works for $\mathcal{X}(M)$ in place of $\mathcal{X}_{D}(M)$ by replacing $d_{D}$ with $d(A, B)=\min \left(1, d_{D}(A, B)\right)$ if $A, B \in \mathcal{X}_{D}(M)$ for some $D$, and $d(A, B)=1$ otherwise.

Remark 3.3. The proof of Lemma 3.1 goes through as written if we give $\mathcal{X}(M)$ the $C^{k}$ topology, where $k$ is any nonnegative integer.

Remark 3.4. Let $G$ be any group of isometries of $\mathbb{R}^{2}$ that contains a reflection $r$ in the $y$-axis, as well as a (nontrivial) rotation about the origin. Let us justify the claim made before Question 1.8 that there is no Hausdorff continuous $G$-equivariant center defined on the set of 2-disks in $\mathcal{X}\left(\mathbb{R}^{2}\right)$. The unit disk $D^{2} \subset \mathbb{R}^{2}$ is Hausdorff close to a smoothly embedded 2-disk $D$ obtained by removing from $D^{2}$ a small $r$-invariant neighborhood of the segment $\left\{(0, y) \in D^{2}: y \geq-\frac{1}{2}\right\}$. Since $G$ contains $r$, any $G$-equivariant center of $D$ is contained in $\left\{(0, y) \in D^{2}: y<-\frac{1}{2}\right\}$, while the $G$-invariant center of $D^{2}$ is the origin because $G$ contains a rotation.

\section{Proof of Theorem 1.1}

In this section $M, G$ are as in Section 1. Thus $M^{G}$ is a smooth submanifold of $M$, and if $D$ is a compact subset of $M$, then $G_{D}$ is a compact subgroup of $G$. Also $D^{G_{D}}=D \cap M^{G_{D}}$. By small we mean close to the identity.

Lemma 4.1. Any $D \in \mathcal{X}(M)$ has a compatible smooth atlas $\alpha$ such that the given $G_{D}$-action on $(D, \alpha)$ can be $C^{1}$ approximated by a smooth $G_{D}$-action, and moreover, these two actions are conjugate by a $C^{1}$ diffeomorphism that is $C^{1}$ close to the identity. 
Proof. The existence of a compatible smooth atlas is proved in [Hir94, Theorem 3.6, Chapter 2]. If $D$ is a closed manifold, the other claims are contained in [Pal70, Theorem $\mathrm{C}$ ]. If $D$ has boundary, we first double the given $C^{1}$ actions of $G_{D}$ along $\partial D$. To equivariantly smooth the double at $\partial D$ we may have to adjust the action, conjugating it by a small $C^{1}$ isotopy near $\partial D$. Then we apply [Pal70, Theorem $\mathrm{C}]$ to the double to approximate the $C^{1}$ action by a $C^{1}$ equivalent smooth action. This action stabilizes a copy of $D$ that is $C^{1}$ close to $D$, so after a conjugation by a small smooth diffeomorphism it can be made to stabilize $D$ inside the double.

Lemma 4.2. Let $D \in \mathcal{X}(M)$ and suppose $D^{G_{D}} \neq \emptyset$. Then $D^{G_{D}}$ is a properly embedded $C^{1}$ submanifold of $D$ that intersects the relative interior of $D$ and is transverse to $\partial D$.

Proof. In the compatible atlas of Lemma 4.1 the $G_{D}$-action on $D$ is near a smooth action, and the two actions are conjugate by a small $C^{1}$ diffeomorphism. Let $\phi$ denote the smooth $G_{D}$-action on the double of $D$, and fix a Riemannian metric invariant under $\phi$. Its fixed point set is a smooth compact boundaryless submanifold which is totally geodesic in the metric. Since $\partial D$ is $\phi$-invariant if $\phi$ fixes $x \in \partial D$, then it also fixes every point in a convex neighborhood of $x$ that lies on the geodesic through $x$ that is orthogonal to $\partial D$. By assumption $G_{D}$ fixes a point of $D$, and hence so does $\phi$. It follows that the fixed point of $\phi$ intersects the relative interior of $D$ and is transverse to $\partial D$. Hence the same is true for the given $G_{D}$ action on the double, and the claim follows.

Remark 4.3. Lemma 4.2 is immediate by transversality if $\operatorname{dim}(D)=\operatorname{dim}(M)$. In general, the intersection of $\partial D$ and $D^{G_{D}}$ is not transverse, e.g., a round disk $D$ in the $x y$-plane in $\mathbb{R}^{3}$ is invariant under rotation by $\pi$ about the $x$-axis, which would be disjoint from $\partial D$ if they met transversely.

We partition $\mathcal{X}(M)$ as $\bigcup_{k, l} \mathcal{D}^{k, l}$ where $\mathcal{D}^{k, l}(M)$ is the set of all $D \in \mathcal{X}(M)$ such that $D^{G_{D}}$ is a $k$-dimensional manifold with $l$ connected components.

Remark 4.4. Even when $D$ is a disk, the manifold $D^{G_{D}}$ may have any finite number of components, e.g., if $G$ is connected and nonabelian and $F$ is any finite CW complex, then there is a smooth $G$-action on some high-dimensional disk $D$ such that $D^{G_{D}}$ is homotopy equivalent to $F$, see [Oli76, Theorems 3 and 5]. On the other hand, we shall see in Appendix A that if $D$ is a disk of dimension $\leq 4$, then $D^{G_{D}}$ is acyclic, and in particular, connected.

Henceforth, we need the following notion of a tubular neighborhood of a $C^{1}$ submanifold $N$ of a Riemannian manifold $M$. By [Hir94, Thm. 3.6, Chap 2] there is a $C^{1}$ diffeomorphism $\phi: M \rightarrow M$ such that $\widetilde{N}:=\phi(N)$ is a $C^{\infty}$ submanifold of $M$. If $\widetilde{U}$ is a Riemannian tubular neighborhood of $\widetilde{N}$ (produced via the normal exponential map), then we call $U:=\phi^{-1}(\widetilde{U})$ a tubular neighborhood of $N$, and furthermore, if $\tilde{\pi}: \widetilde{U} \rightarrow \widetilde{N}$ is the nearest point projection, we call $\pi:=\phi^{-1} \circ \tilde{\pi} \circ \phi: U \rightarrow N$ the normal bundle projection of $U$. 
Lemma 4.5. The map $\mathcal{D}^{k, l}(M) \rightarrow \mathcal{X}(M)$ given by $D \rightarrow D^{G_{D}}$ is continuous.

Proof. Fix any $J \in \mathcal{D}^{k, l}(M)$ and prove continuity at $J$. By Lemma 2.3(vi) there is a neighborhood $U$ of $J$ in $\mathcal{D}^{k, l}(M)$ such that $G_{D}$ can be conjugated into $G_{J}$ by a small element of $G$, and since such conjugation results in only small $C^{1}$ changes of $D$ and $D^{G_{D}}$ we may assume that $G_{D} \leq G_{J}$. Thus both $D$ and $J$ are invariant under $H=G_{D}$.

After possibly shrinking $U$ further we can find a $C^{1}$ diffeomorphism $\psi$ with $\psi(D)=$ $J$ such that $\psi$ is supported in a small neighborhood of $J$. (To find $\psi$ we first isotope $\partial D$ to $\partial J$ in the the tubular neighborhood $\partial J$, then extend $J$ to a boundaryless embedded $C^{1}$ submanifold, use its tubular neighborhood to isotope $D$ to $J$ relative boundary, and finally extend the isotopy to an ambient one). Thus $\psi H \psi^{-1}$ and $H$ are two $C^{1}$ close $H$-actions on $J$. By Lemma 4.1 these actions are $C^{1}$ diffeomorphic via a small diffeomorphism.

By Lemma 4.2 the fixed point sets of the actions of $\psi H \psi^{-1}, H, G_{J}$ on $J$ are compact properly embedded $k$-dimensional $C^{1}$ submanifolds of $J$ with $l$ connected components. Any proper embedding of compact $k$-manifolds with the same number of components is surjective, so since $H \leq G_{J}$ the fixed point sets of $H, G_{J}$ in $J$ coincide. Thus $J^{G_{J}}$ is $C^{1}$ close to the fixed point set of $\psi H \psi^{-1}$ which is $D^{G_{D}}$.

Lemma 4.6. If $B$ is a compact $C^{1}$ submanifold of a manifold $F$, then there are two nested tubular neighborhoods $T \subset T_{2}$ of $\partial B$ in $F$ and a $C^{1}$ self-map $q_{B}$ of $F$ that is the identity outside $T_{2}$, and that equals the normal bundle projection on $T$.

Proof. Fix a tubular neighborhood $T_{2}$ of $\partial B$ in $F$, and identify it with $[-2,2] \times \partial B$ where $\partial B$ corresponds to $\{0\} \times \partial B$. Let $T$ be the subset of $T_{2}$ corresponding under the identification to $[-1,1] \times \partial B$. Fix a smooth non-decreasing self-map $\tau$ of $[-2,2]$ that vanishes on $[-1,1]$ and equals the identity near the endpoints $-2,2$. Then the self-map of $[-2,2] \times \partial B$ given by $(t, z) \rightarrow(\tau(t), z)$ interpolates between the identity near the boundary and the projection $[-1,1] \times \partial B \rightarrow\{0\} \times \partial B$.

Let $\mathcal{E}^{k, l}(M)$ be the subspace of $\mathcal{D}^{k, l}(M) \times M$ consisting of all the pairs $(D, u)$ with $D \in \mathcal{D}^{k, l}(M)$ and $u \in D^{G_{D}}$. Let $\pi: \mathcal{E}^{k, l}(M) \rightarrow \mathcal{D}^{k, l}(M)$ be the coordinate projection, i.e., $\pi(D, u)=D$.

Lemma 4.7. The map $\pi: \mathcal{E}^{k, l}(M) \rightarrow \mathcal{D}^{k, l}(M)$ is a locally trivial bundle.

Proof. Fix $J \in \mathcal{D}^{k, l}(M)$, set $B=J^{G_{J}}$, and extend $B$ to a $k$-dimensional boundaryless $C^{1}$ submanifold $F$ of $M$. Let $q_{F}$ be a projection of a tubular neighborhood of $F$ in $M$. For such $B, F$ let $q_{B}$ and $T$ be as in Lemma 4.6. Let $q=q_{B} \circ q_{F}$. Using Lemma 4.5 we can assume that $D$ is so close to $J$ that $q_{F}\left(D^{G_{D}}\right) \subset T$ and $q_{B}$ restricts to a $C^{1}$ diffeomorphism of $q_{F}\left(D^{G_{D}}\right)$ onto $B$. The map $(D, u) \rightarrow(D, q(u))$ is the desired local trivialization where $u \in D^{G_{D}}$.

Remark 4.8. The homeomorphism type of the fiber $\pi^{-1}(D)$ may depend on $D$. 
The group $G$ acts on $\mathcal{E}^{k, l}(M)$ diagonally, i.e., $g(D, u)=(g D, g u)$. In the following commutative diagram

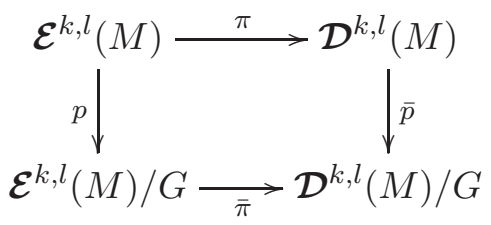

the vertical arrows are the $G$-orbit maps, and $\bar{\pi}$ sends the $G$-orbit of $(D, u)$ to the $G$-orbit of $D$.

By Remark 3.2 the $G$-spaces $\mathcal{D}^{k, l}(M)$ and $\mathcal{E}^{k, l}(M)$ are Palais-proper because the property is clearly inherited by invariant subspaces and preimages under equivariant maps. Also $\mathcal{E}^{k, l}(M)$ has a $G$-invariant metric induced by the $G$-invariant metrics on the factors of $\mathcal{D}^{k, l}(M) \times M$. The following result is a key observation of this paper, which reduces to Lemma 4.7 when $G$ is trivial.

Lemma 4.10. $\bar{\pi}: \mathcal{E}^{k, l}(M) / G \rightarrow \mathcal{D}^{k, l}(M) / G$ is a locally trivial fiber bundle.

Proof. Let us first sketch the proof. The $G$-action permutes the fibers of $\pi$ which are of the form $\pi^{-1}(y)=\left\{(y, u): u \in y^{G_{y}}\right\}$. Moreover, each fiber projects homeomorphically to $\mathcal{E}^{k, l}(M) / G$ because the fiber is compact and the projection $p$ is injective on it: If $g(y, u)=(y, v)$ with $u, v \in y^{G_{y}}$, then $g \in G_{y}$ and $g u=v$. Hence $g u=u$ implies $u=v$. To establish local triviality we analyze the structure of the orbit spaces via local slices. Using that $G_{y} \leq G_{x}$ for every $y$ in a $G_{x}$-slice we show that the local trivialization of Lemma 4.7 can be made $G_{x}$-equivariant, so it passes to the $G_{x}$-quotients of the slices, yielding a local trivialization of $\bar{\pi}$.

Let us make this sketch rigorous. Set $X=\mathcal{D}^{k, l}(M)$. Fix $x \in X$ and set $H=G_{x}$. Let $S$ be a $H$-slice at $x$. Thus $G$ fixes $\pi^{-1}(x)$ pointwise, and hence, Lemma 2.1 implies that $E=\pi^{-1}(S)$ is an $H$-slice at any point of $\pi^{-1}(x)$.

Set $\bar{E}=p(E)$ and $\bar{S}=\bar{p}(S)$. Since $S$ is a slice, $\bar{S}$ is a neighborhood of $\bar{p}(x)$, and it suffices to show that $\bar{\pi}$ is a locally trivial bundle over $\bar{S}$.

Let us show that $\bar{E}=\bar{\pi}^{-1}(\bar{S})$. One inclusion follows from a diagram chase: If $z \in p\left(\pi^{-1}(S)\right)$, then $\bar{\pi}(z) \in(\bar{\pi} \circ p)\left(\pi^{-1}(S)\right)=(\bar{p} \circ \pi)\left(\pi^{-1}(S)\right)=p(S)$. Conversely, if $z \in \bar{\pi}^{-1}(\bar{S})$, then $\bar{\pi}(z)=G y$ for some $y \in S$. Also $z$ is the $G$-orbit of some $(a, v)$ with $a \in X, v \in a^{G_{a}}$, and hence $\bar{\pi}(z)$ is the $G$-orbit of $\pi(a, v)=a$. Thus $a=g y$ for some $g \in G$. Hence $z$ is the $G$-orbit of $\left(y, g^{-1} v\right)$, and moreover, $g^{-1} v \in y^{G_{y}}$. Thus $\left(y, g^{-1} v\right) \in E$, and so $z \in \bar{E}$.

Since $E$ and $S$ are $H$-slices, the inclusion induced maps $E / H \rightarrow \bar{E}, S / H \rightarrow \bar{S}$ are homeomorphisms, so we can identify the map $\bar{\pi}: \bar{E} \rightarrow \bar{S}$ with the map $E / H \rightarrow S / H$ also induced by $\pi$.

Set $B=x^{H}$ and note that $B$ is contained in $M^{H}$, which is a boundaryless properly embedded smooth submanifold of $M$. We can extend $B$ to a $k$-dimensional 
boundaryless $C^{1}$ submanifold $F$ of $M^{H}$. Fix an $H$-invariant tubular neighborhood of $F$ in $M$, and let $q_{F}$ be an $H$-equivariant projection of the neighborhood onto $M$ (obtained, e.g., as the nearest point projection of some smooth $H$-invariant Riemannian metric on $M)$.

For such $B, F$ let $q_{B}$ and $T$ be as in Lemma 4.6. Now Lemma 4.5 implies that by making $S$ smaller we can assume that any $y \in S$ satisfies $q_{F}\left(y^{G_{y}}\right) \subset T$ and $q_{B}$ restricts to a $C^{1}$ diffeomorphism of $q_{F}\left(y^{G_{y}}\right)$ onto $B$. (Lemma 2.3(v) allows us to make $S$ arbitrary small.) Then for each $y \in S$ the composite $q=q_{B} \circ q_{F}$ restricts to a $C^{1}$-diffeomorphism of $y^{G_{y}}$ onto $B$.

For every $y \in S$ we have $G_{y} \leq H$, and hence $M^{G_{y}} \supseteq M^{H} \supseteq F$. In particular, if $z \in y^{G_{y}}$ and $y \in S$, then $q(h z)=q(z)$ because $q$ is $H$-equivariant and $H$ acts trivially on the image of $q$.

The map $\phi: E \rightarrow S \times B$ given by $(y, u) \rightarrow(y, q(u))$ is $H$-equivariant because it sends $h(y, u)=(h y, h u)$ to $(h y, q(h u))=(h y, q(u))=(h y, h q(u))=h(y, q(y))$, Also $\phi$ is a homeomorphism whose inverse sends $(y, v)$ to $\left(y,\left(\left.q\right|_{y^{G_{y}}}\right)^{-1}(v)\right)$. Note that $H$ acts trivially on the $B$-factor. So $\phi$ descends to a homeomorphism of the $H$-quotients $E / H \rightarrow S / H \times B$ which gives a desired local trivialization.

Let $\mathcal{D}^{k}(M)$ be the subspace of $D \in \mathcal{X}(M)$ such that $D^{G_{D}}$ is contractible and $k$-dimensional. Set $\mathcal{E}^{k}(M)=\left\{(D, u): D \in \mathcal{D}^{k}(M), u \in D^{G_{D}}\right\}$. Note that $\mathcal{D}^{k}(M), \mathcal{E}^{k}(M)$ are $G$-invariant subsets of $\mathcal{D}^{k, 1}(M), \mathcal{E}^{k, 1}(M)$, respectively, and moreover, $\mathcal{E}^{k}(M)$ is the $\pi$-preimage of $\mathcal{D}^{k}(M)$. Thus $\pi: \mathcal{E}^{k}(M) \rightarrow \mathcal{D}^{k}(M)$ and $\bar{\pi}: \mathcal{E}^{k}(M) / G \rightarrow \mathcal{D}^{k}(M) / G$ are locally trivial fiber bundles.

Lemma 4.11. If $A$ is a closed $G$-invariant subset of $\mathcal{D}^{k}(M)$, then every continuous $G$-equivariant section $s_{A}: A \rightarrow \mathcal{E}^{k}(M)$ of $\pi$ over $A$ extends to a continuous $G$-equivariant section $s$ of $\pi$ over $\mathcal{D}^{k}(M)$. Moreover, if $s_{A}$ takes values in the interiors of the fibers, then one can choose $s$ with the same property.

Proof. By $G$-equivariance, $s_{A}$ descends to a continuous map $\bar{s}_{A}: A / G \rightarrow \mathcal{E}^{k, l}(M) / G$ that takes the $G$-orbit of $x \in A$ to the $G$-orbit of $s(x)$, i.e., $\bar{s}_{A}(\bar{p}(x))=p\left(s_{A}(x)\right)$. The following section extension property can be found in [Pal66, Theorem 9]: Given any locally trivial fiber bundle whose base is metrizable and fiber is an absolute retract, any section of the bundle defined on a closed subset can be extended to the whole base. In our case the fiber is either a compact contractible manifold with boundary, or its interior, which are absolute retracts.

By definition of the quotient topology, $A / G$ is closed in $\mathcal{D}^{k, l}(M) / G$. Thus $\bar{s}_{A}$ extends to a continuous section $\bar{s}$ of $\bar{\pi}$. If $s_{A}$ takes values in the interiors of the fibers, then so does $\bar{s}_{A}$, and hence it can be extended to $\bar{s}$ with the same property. Now define $s: \mathcal{D}^{k, l}(M) \rightarrow \mathcal{E}^{k, l}(M)$ by letting $s(x)$ be the intersection of the fiber $\pi^{-1}(x)$ and the $G$-orbit $p^{-1}(\bar{s}(\bar{p}(x)))$, see the diagram (4.9). Let us check that $s$ has the claimed properties. 
The intersection consists of a single point, indeed, if $z, g z$ are in the intersection, then $\pi(z)=x=\pi(g z)=g \pi(z)$ and hence $g$ is in the isotropy subgroup of $z$, which fixes $\pi^{-1}(\pi(z))$ pointwise so that $g z=z$. If $a \in A$, then $s(a)$ and $s_{A}(a)$ are points of $\pi^{-1}(a)$ that are mapped by $p$ to $\bar{s}(\bar{p}(a))=\bar{s}_{A}(\bar{p}(a))$, and since such a point is unique, $s$ extends $s_{A}$.

By construction $\left.\pi(s(x)) \subseteq \pi\left(\pi^{-1}(x)\right)\right)=\{x\}$, so $s$ is a section. The map $s$ is $G$-equivariant because $s(g x)$ is the intersection of $\pi^{-1}(g x)=g \pi^{-1}(x)$ and $p^{-1}(\bar{s}(\bar{p}(g x)))=p^{-1}(\bar{s}(\bar{p}(x)))=g\left(p^{-1}(\bar{s}(\bar{p}(x)))\right)$, which equals $g s(x)$.

We write $u \approx v$ to indicate that $u, v$ are close to each other. To prove continuity of $s$ write $s(x)=(x, c)$ and $s(y)=(y, d)$, where $c \in x, d \in y$, assume $x \approx y$, and try to show that $c \approx d$. From $x \approx y$ we get closeness of the the $G$-orbits $p^{-1}\left(\bar{s}(\bar{p}(x)), p^{-1}(\bar{s}(\bar{p}(y))\right.$ of $s(x), s(y)$, respectively. Hence there is $g \in G$ such that $g s(x) \approx s(y)$, or equivalently, $g x \approx y$ and $g c \approx d$. From $x \approx y$ we get $g x \approx g y$, and hence $x \approx y \approx g x \approx g y$. Then $g$ is close to some elements of the isotropy subgroups of $x, y$, which fix the fibers $\pi^{-1}(x), \pi^{-1}(y)$ pointwise. Hence $c \approx g c$ and $d \approx g d$ which together with $g c \approx d$ implies $c \approx d$.

The above proof also yields the following observation:

Lemma 4.12. If $A$ is a closed $G$-invariant subset of $\mathcal{D}^{k}(M)$, then every $G$-equivariant section $\bar{s}_{A}: A / G \rightarrow \mathcal{E}^{k, l}(M) / G$ extends to a continuous section $\bar{s}$ of $\bar{\pi}$ over $\mathcal{D}^{k}(M) / G$ which lifts to $G$-equivariant section of $\pi$ over $\mathcal{D}^{k}(M)$. Moreover, if $\bar{s}_{A}$ takes values in the interiors of the fibers, then one can choose $s$ with the same property.

Set $\mathcal{D}(M)=\bigcup_{k=0}^{n} \mathcal{D}^{k}(M)$. We are now ready to prove Theorem 1.1.

Theorem 4.13. For any $G$-invariant closed subset $Z$ of $\mathcal{X}(M)$ every $G$-equivariant center $\mathfrak{z}: Z \rightarrow M$ extends to a $G$-equivariant center $\mathfrak{c}: \mathcal{D}(M) \rightarrow M$.

Proof. Given a nonnegative integer $m$ let $\mathcal{S}^{m}=\bigcup_{i \leq m} \mathcal{D}^{i}$. Note that $\mathcal{S}^{m}$ is closed in $\mathcal{D}(M)$ for if $D_{i} \rightarrow D$, then $G_{D}$ contains a conjugate of $G_{D_{i}}$ by Lemma 2.3(vi) and, in particular, if each $D_{i}$ lies in $\mathcal{S}^{m}$, then so does $D$.

We proceed by induction on the dimension of $D^{G_{D}}$. Let $l$ be the smallest integer with $\mathcal{S}^{l} \neq \emptyset$. Use Lemma 4.12 with $A=Z \cap \mathcal{S}^{l}$ to define a $G$-equivariant center $\mathfrak{c}: \mathcal{S}^{l} \rightarrow M$ that extends $\mathfrak{z}$. Inductively, we suppose that a map $\mathfrak{c}$ with claimed properties is defined on $\mathcal{S}^{k}$, and hence on $\mathcal{Z}^{k}=\mathcal{S}^{k} \cup\left(Z \cap \mathcal{S}^{k+1}\right)$, and then try to extend it to $\mathcal{S}^{k+1}$.

To this end fix a smooth $G$-equivariant embedding of $\tau: M \rightarrow \mathcal{H}$ where $\mathcal{H}$ is a Hilbert space equipped with the orthogonal $G$-action [Kan94, Theorem 0.1]. The latter means that each element of $g$ is continuous, linear, and preserves the inner product. Then the usual Riemannian open tubular neighborhood $T_{\tau}$ of $\tau$ is $G$ invariant and its projection $p_{\tau}: T_{\tau} \rightarrow M$ is $G$-equivariant [Kan07, Theorem 5.1]. 
In [Ant05, Corollary 3.5 and Example 4.1] one finds a general extension result for $G$-maps from a Palais-proper space $X$ with a $G$-invariant metric to any locally convex linear $G$-space, such as $\mathcal{H}$. It implies that for any closed $G$-invariant subset $A$ of $X$ every $G$-map $A \rightarrow \mathcal{H}$ can be extended to a $G$-map $X \rightarrow \mathcal{H}$. For this result the $G$-action on $\mathcal{H}$ must be linear, continuous, and every compact subgroup of $G$ has to fix a point of $\mathcal{H}$. The latter is true for orthogonal actions of compact groups on $\mathcal{H}$, and in fact, any isometric group action with a bounded orbit on a $\mathrm{CAT}(0)$ space fixes the circumcenter of the orbit, and in a $\mathrm{CAT}(0)$ space, such as $\mathcal{H}$, every bounded set has a unique circumcenter [BH99, Proposition II.2.7].

Thus $\mathfrak{c}: \mathcal{Z}^{k} \rightarrow M \subset \mathcal{H}$ can be extended to a $G$-map from $\tilde{\mathfrak{c}}: \mathcal{S}^{k+1} \rightarrow \mathcal{H}$. Set $O_{\tau}=\tilde{\mathfrak{c}}^{-1}\left(T_{\tau}\right)$ and $\bar{c}=\left.p_{\tau} \circ \tilde{\mathfrak{c}}\right|_{O_{\tau}}$. Thus $\overline{\mathfrak{c}}: O_{\tau} \rightarrow M$ is a $G$-map that extends $\mathfrak{c}$.

Let us show that there is a closed neighborhood $V$ of $\mathcal{Z}^{k}$ in $O_{\tau}$ on which $\bar{c}(D) \in$ $\operatorname{Int}(D)$. To this end fix a $G$-invariant metric $d$ on $\mathcal{D}$ (using the metric of Remark 3.2 in each $\mathcal{D}^{k}(M)$ and setting the distance between points of different $\mathcal{D}^{k}(M)$ to be $1)$. Since $\overline{\mathfrak{c}}$ is continuous for any $D \in \mathcal{D}$ with $\overline{\mathfrak{c}}(D) \in \operatorname{Int}(D)$ there is $\varepsilon_{D}>0$ such that $\overline{\mathfrak{c}}(I) \in \operatorname{Int}(I)$ for every $I$ in the $\varepsilon_{D}$-ball centered at $D$. We can use the same $\varepsilon_{D}$ for every $D$ in the same $G$-orbit. The intersection with $\mathcal{S}^{k+1}$ of the union of all such balls is a $G$-invariant neighborhood $U$ of $\mathcal{Z}^{k}$ in $\mathcal{S}^{k+1}$, and $\left.\overline{\mathfrak{c}}\right|_{U}(D) \in \operatorname{Int}(D)$. Finally, $U$ contains a closed $G$-invariant neighborhood $V$ of $\mathcal{Z}^{k}$, e.g., let $V$ be the $f$-preimage of $\left[0, \frac{1}{2}\right]$ where

$$
f(x)=\frac{d\left(x, \mathcal{Z}^{k}\right)}{d\left(x, \mathcal{Z}^{k}\right)+d\left(x, \mathcal{S}^{k+1} \backslash U\right)} .
$$

It remains to enlarge $V$ to $\mathcal{S}^{k+1}$. The restriction of $\overline{\mathfrak{c}}$ to $V_{0}=V \cap \mathcal{D}^{k+1}$ is a $G$-equivariant section of the bundle $\mathcal{E}^{k+1} \rightarrow \mathcal{D}^{k+1}$ over $V_{0}$. Applying Lemma 4.11 to the bundle whose fibers are $\operatorname{Int}\left(D^{G_{D}}\right)$, we extend $\left.\overline{\mathfrak{c}}\right|_{V_{0}}$ to a $G$-equivariant section $\mathfrak{c}_{0}$ over $\mathcal{D}^{k+1}$. Since $\mathcal{D}^{k+1}, \operatorname{Int}(V)$ are open in $\mathcal{S}^{k+1}$ and $\mathfrak{c}_{0},\left.\overline{\mathfrak{c}}\right|_{\operatorname{Int}(V)}$ agree on $\mathcal{D}^{k+1} \cap \operatorname{Int}(V)$, the maps define a $G$-map $\mathcal{S}^{k+1} \rightarrow M$ that takes each $D$ to a point in $\operatorname{Int}(D)$. This completes the induction step and proves the theorem.

Remark 4.14. The above proof reveals that Theorem 1.1 is a formal consequence of Lemma 4.7, and hence if the lemma is true for the $C^{0}$ topology on $\mathcal{X}(M)$, then the answer to Question 1.8 is affirmative.

Proof of Proposition 1.6. As discussed before the statement of the corollary, there exists an orthogonal $A_{5}$-action on $\mathbb{R}^{r}$ that has no fixed points on an $A_{5}$-invariant smoothly embedded 6 -disk in $\mathbb{R}^{r}$. Denote the 6 -disk by $\Delta$, consider the product of $\Delta$ and the $(n-6)$-disk with the trivial $A_{5}$-action, and smooth corners. The result is a smoothly embedded $A_{5}$-invariant $n$-disk in $\mathbb{R}^{n+r-6}$ on which $A_{5}$ acts without fixed points. Composing with the standard inclusion $\mathbb{R}^{n+r-6} \subset \mathbb{R}^{m}$ we can think of the $n$-disk as sitting in $\mathbb{R}^{m}$, where we let $A_{5}$ act trivially on the orthogonal complement of $\mathbb{R}^{r+n-6}$ in $\mathbb{R}^{m}$. This gives the desired claim when $m \geq n+r-6 \geq r$. 
For the other case consider the inclusion $\mathbb{R}^{r} \subset \mathbb{R}^{n}$, where $A_{5}$ acts trivially on the orthogonal complement of $\mathbb{R}^{r}$ in $\mathbb{R}^{n}$. Let $\Delta^{\prime}$ be an $A_{5}$-invariant smooth tubular neighborhood of $\Delta$ in $\mathbb{R}^{n}$ on which the $A_{5}$-action is fixed point free. Note that $\Delta^{\prime}$ is an embedded $n$-disk in $\mathbb{R}^{n}$, so composing with the inclusion $\mathbb{R}^{n} \subset \mathbb{R}^{m}$ gives the claim when $m \geq n \geq r$.

\section{Appendix A. Compact Lie Group Actions on Low Dimensional Disks}

The results of this appendix are surely known to experts but we could not find them in the literature.

It was shown by B. Kerékjártó that any compact topological group action on a 2-disk is equivalent to a linear action, see [Kol06] and references therein. More precisely, cone off the boundary of the $G$-action on $D^{2}$, use [Kol06] to conclude that the resulting action on $S^{2}$ is topologically equivalent to an action by a subgroup of $O(3)$, which is actually in $O(2)$ since it has a fixed point, and finally restrict the equivalence to $D^{2}$.

For a smooth compact Lie group action, the uniformization gives an alternative route: Find a $G$-invariant Riemannian metric on the interior, and map it conformally to the standard hyperbolic disk so that the Lie group becomes a compact group of hyperbolic isometries, and hence after conjugation the standard $O(2)$. The same reasoning gives linearity of smooth actions on the closed interval, whose isometry group in any metric is $O(1)$.

It was shown in [KS92, Theorem B] that any smooth action of a compact Lie group on the 3 -disk is smoothly equivalent to a linear action.

Lemma A.1. If $G$ is a nontrivial finite group that acts smoothly and effectively on the 4-disk $D$, then either $D^{G}$ is a disk of dimension $\leq 2$, or $G$ is an order two group generated by an orientation-reversing involution and $D^{G}$ is a compact acyclic 3-manifold.

Proof. Any smooth finite group action on a 4-disk has a fixed point [BKS90, Theorem II.2]. Thus $D^{G}$ is a compact smooth properly embedded submanifold of $D$. Let $S$ be the double of $D$ along $\partial D$ equipped with the smooth $G$-action. Thus $S$ is a smooth homotopy 4 -sphere and $S^{G}$ is nonempty.

The fixed point set of any smooth (or, more generally, locally linear) orientationpreserving action of a finite group on a homology 4-sphere is either empty or homeomorphic to a sphere [DM89, Theorem 2.1]. Let $H \leq G$ be the subgroup of the orientation-preserving elements. The group $G / H$ has order at most two, because $H$ is the kernel of the $G$-action on $H_{4}(D, \partial D ; \mathbb{Z}) \cong \mathbb{Z}$.

Thus $S^{H}$ is a closed smooth submanifold of $S$ that is homeomorphic to a sphere of dimension $l \in[0,4]$. Since $H$ preserves orientation, we have $l \neq 3$ else $H$ would have to act nontrivially on a one-dimensional fiber of the normal bundle to $D^{G}$. If 
$l \leq 2$, then $D^{H}$ is homeomorphic to the $l$-disk, the only manifold whose double is the $l$-sphere. Since $D^{G}$ is the fixed point set of the $G / H$-action on $D^{H}$ we conclude for $l \leq 2$ that $G / H$ acts linearly on $D^{G}$, and hence $D^{G}$ is a subdisk of $D^{H}$ as claimed.

It remains to consider the case $l=4$ where $H$ is trivial and $G$ is generated by an orientation-reversing involution of $D$. Then $D^{G}$ is a homology disk [Bre72, Theorem III.5.2] of dimension $l \in[0,3]$. The $G$-action in a fiber of the normal bundle to $D^{G}$ only fixes the origin and reverses the orientation, hence $l$ is odd. If $l=1$, then $D^{G}$ is a one-dimensional disk, as claimed, and we are left with the exceptional case $l=3$ where $D^{H}$ is acyclic.

Remark A.2. Any compact acyclic 3-dimensional smooth submanifold of $S^{3}$ is homeomorphic to $D^{3}$ because its boundary is a homology 2 -sphere, and hence is homeomorphic to $S^{2}$, so that the Schoenflies theorem applies [Bro60]. In particular, if $M$ and $G$ are as in Corollary 1.5 and $D$ is a $C^{1}$ embedded 4-disk in $M$, then $D^{G_{D}}$ is homeomorphic to a disk.

Lemma A.3. If $G$ is an infinite compact Lie group that acts smoothly and effectively on the 4 -disk $D$, then $D^{G}$ is a disk of dimension $\leq 2$.

Proof. If the claim is true for the connected component $G_{0}$ of $G$, then it is true for $G$ because if $G_{0}$ fixes a disk $F$ of dimension $\leq 2$, then $G / G_{0}$ acts on $F$. Since any compact Lie group action on a disk of dimension $\leq 2$ is equivalent to a linear action, $G / G_{0}$ fixes a subdisk of $F$ of dimension $\leq 2$, which is then fixed by $G$.

Thus we may assume that $G$ is connected. By the structure theory of compact Lie groups there is a surjective homomorphism $T \times \bar{G} \rightarrow G$ with finite kernel where $T$ is a torus and $\bar{G}$ is a semisimple connected compact Lie group. The resulting (possibly ineffective) $T$-action on $D^{4}$ has a fixed point set $D^{T}$ which is an integral homology disk of dimension 0,2 , or 4 , see [Bre72, Theorem III.10.3], and in particular it is non-empty. If $T$ is nontrivial, then $D^{T}$ is a nowhere dense [Bre72, Theorem III.9.5] smooth submanifold of $D$, and so $\operatorname{dim}\left(D^{T}\right)<4$. Thus $D^{T}$ is diffeomorphic to a disk of dimension $\leq 2$. Since $\bar{G}$ normalizes $T$, it acts on $D^{T}$, and the argument of the previous paragraph applies to show that $G$ fixes a disk of dimension $\leq 2$.

Thus we may assume that $G$ is semisimple. The principal orbit $G / H$ cannot be a point because then $G$ fixes an open subset of $D$ [Bre72, Theorem IV.3.1], and cannot be a circle as, e.g., in the homotopy sequence of the bundle $G \rightarrow G / H$ the group $\pi_{1}(G / H)$ sits between finite groups $\pi_{1}(G), \pi_{0}(H)$. Also $G / H$ is a closed manifold embedded in $D^{4}$, so its dimension is at most 3. Thus $G / H$ has codimension 1 or 2. Then [Bre72, Theorem IV.8.1 and Theorem IV.8.5] imply that the $G$-action on $\operatorname{Int}(D)$ is equivalent to an orthogonal action $\mathbb{R}^{4}$, so its fixed point set is a linear subspace $V$, and in particular is nonempty. Since $D^{G}$ is a smooth submanifold that is transverse to $\partial D$, we get $\operatorname{Int}\left(D^{G}\right)=V$. Thus

$$
0 \leq \operatorname{dim}\left(D^{G}\right)=\operatorname{dim}(V) \leq \operatorname{dim}(\operatorname{Int}(D) / G)=4-\operatorname{dim}(G / H) \leq 2
$$


and therefore $D^{G}$ is a disk of dimension $\leq 2$.

Lemma A.4. There is a smooth involution on $D^{4}$ whose fixed point set is an acyclic non-simply connected 3 -manifold with boundary that is properly embedded in $D^{4}$.

Proof. In [Maz61, Poe60] one finds a smooth compact contractible 4-manifold $C$ such that $\partial C$ is not simply-connected while the double $D C$ of $C$ along $\partial C$ is diffeomorphic to $S^{4}$. Let $\iota$ be the doubling involution. Removing a small $\iota$-invariant open ball $B$ centered at $\partial C$ yields a $\iota$-invariant copy of $D^{4}$ that is the double of $C \backslash B$ along $\partial C \backslash B$. Here $\iota$ permutes two copies of $C \backslash B$ and fixes $\partial C \backslash B$ pointwise. Finally, since $\partial C$ is a non-simply connected homology sphere, $\partial C \backslash B$ is a compact acyclic non-simply connected 3-manifold.

Here is an example of a 5 -disk $D$ in $\mathbb{R}^{5}$ such that $G_{D}$ is generated by a linear involution and $D^{G_{D}}$ is not contractible.

Lemma A.5. If $r$ is the reflection in the equator of $S^{5}$ and $k \in\{4,5\}$, then $S^{5}$ contains a smoothly embedded $r$-invariant copy of $D^{k}$ which transversely intersects the equator in an acyclic non-simply connected $(k-1)$-manifold with boundary.

Proof. In the notations of Lemma A.4 consider a proper embedding of $C$ into $D^{5}$ (e.g., obtained by isotoping $C \subset S^{4}=\partial D^{5}$ into the interior of $D^{5}$ and concatenating the result with the track of isotopy over $\partial C$ ). Removing from $C$ a small $r$-invariant open 4-disk $B$ centered at a point of $\partial C \subset \partial D^{5}$ and then doubling along $\partial D^{5}$ gives an $r$-invariant copy of $D C \backslash B$ of Lemma A.4 in $S^{5}$ that intersects the equator along $\partial C \backslash B$. This covers the case $k=4$. For the case $k=5$ set $I=[-1,1]$ and note that $D C \times I$ embeds as an $r$-invariant tubular neighborhood of $D C$ in $S^{5}$, and after smoothing corners $(D C \backslash B) \times I$ is an $r$-invariant 5 -disk in $S^{5}$ that intersects the equator along $(C \backslash B) \times I$.

\section{ACKNOWLEDGMENTS}

M.G. first learned about the problem of finding a center for planar Jordan domains from Eugenio Calabi in 1995. Also we thank John Etnyre, Ralph Howard, Dan Margalit, and Krzysztof Pawalowski for helpful discussions.

\section{REFERENCES}

[Ant05] S. A. Antonyan, Extending equivariant maps into spaces with convex structure, Topology Appl. 153 (2005), no. 2-3, 261-275.

[BH99] M. R. Bridson and A. Haefliger, Metric spaces of non-positive curvature, Grundlehren der Mathematischen Wissenschaften [Fundamental Principles of Mathematical Sciences], vol. 319, Springer-Verlag, Berlin, 1999.

[BKS90] N. P. Buchdahl, S. Kwasik, and R. Schultz, One fixed point actions on low-dimensional spheres, Invent. Math. 102 (1990), no. 3, 633-662.

[BLP05] M. Boileau, B. Leeb, and J. Porti, Geometrization of 3-dimensional orbifolds, Ann. of Math. (2) 162 (2005), no. 1, 195-290. 
[BM05] A. Bak and M. Morimoto, The dimension of spheres with smooth one fixed point actions, Forum Math. 17 (2005), no. 2, 199-216.

[Bre72] G. E. Bredon, Introduction to compact transformation groups, Academic Press, New York-London, 1972, Pure and Applied Mathematics, Vol. 46.

[Bro60] M. Brown, A proof of the generalized Schoenflies theorem, Bull. Amer. Math. Soc. 66 (1960), 74-76.

[DL09] J. Dinkelbach and B. Leeb, Equivariant Ricci flow with surgery and applications to finite group actions on geometric 3-manifolds, Geom. Topol. 13 (2009), no. 2, 1129-1173.

[DM89] S. De Michelis, The fixed point set of a finite group action on a homology four sphere, Enseign. Math. (2) 35 (1989), no. 1-2, 107-116.

[Hir94] M. W. Hirsch, Differential topology, Graduate Texts in Mathematics, vol. 33, SpringerVerlag, New York, 1994, Corrected reprint of the 1976 original.

[Kan94] M. Kankaanrinta, On embeddings of proper smooth G-manifolds, Math. Scand. 74 (1994), no. 2, 208-214.

[Kan07] _ Equivariant collaring, tubular neighbourhood and gluing theorems for proper Lie group actions, Algebr. Geom. Topol. 7 (2007), 1-27.

[KMT91] M. J. Kaiser, T. L. Morin, and T. B. Trafalis, Centers and invariant points of convex bodies, Applied geometry and discrete mathematics, DIMACS Ser. Discrete Math. Theoret. Comput. Sci., vol. 4, Amer. Math. Soc., Providence, RI, 1991, pp. 367-385.

[KN96] S. Kobayashi and K. Nomizu, Foundations of differential geometry. Vol. I, Wiley Classics Library, John Wiley \& Sons, Inc., New York, 1996, Reprint of the 1963 original, A WileyInterscience Publication.

[Kol06] B. Kolev, Sous-groupes compacts d'homéomorphismes de la sphère, Enseign. Math. (2) 52 (2006), no. 3-4, 193-214.

[KS92] S. Kwasik and R. Schultz, Icosahedral group actions on $\mathbf{R}^{3}$, Invent. Math. 108 (1992), no. 2, 385-402.

[Maz61] B. Mazur, A note on some contractible 4-manifolds, Ann. of Math. (2) 73 (1961), 221228.

[Mic56a] E. Michael, Continuous selections. I, Ann. of Math. (2) 63 (1956), 361-382.

[Mic56b] _ Continuous selections. II, Ann. of Math. (2) 64 (1956), 562-580.

[Mic59] , Paraconvex sets, Math. Scand. 7 (1959), 372-376.

[Mos06] Maria Moszyńska, Selected topics in convex geometry, Birkhäuser Boston, Inc., Boston, MA, 2006, Translated and revised from the 2001 Polish original. MR 2169492

[Oli76] R. Oliver, Smooth compact Lie group actions on disks, Math. Z. 149 (1976), no. 1, 79-96.

[Pal60] R. S. Palais, The classification of $G$-spaces, Mem. Amer. Math. Soc. No. 36, 1960.

[Pal61] On the existence of slices for actions of non-compact Lie groups, Ann. of Math. (2) 73 (1961), 295-323.

[Pal66] Homotopy theory of infinite dimensional manifolds, Topology 5 (1966), 1-16.

[Pal70] — $C^{1}$ actions of compact Lie groups on compact manifolds are $C^{1}$-equivalent to $C^{\infty}$ actions, Amer. J. Math. 92 (1970), 748-760.

[Pix76] C. P. Pixley, Continuously choosing a retraction of a separable metric space onto each of its arcs, Illinois J. Math. 20 (1976), no. 1, 22-29.

[Poe60] V. Poenaru, Les decompositions de l'hypercube en produit topologique, Bull. Soc. Math. France 88 (1960), 113-129.

[RS98] D. Repovš and P. V. Semenov, Continuous selections of multivalued mappings, Mathematics and its Applications, vol. 455, Kluwer Academic Publishers, Dordrecht, 1998.

[RS02] C Continuous selections of multivalued mappings, Recent progress in general topology, II, North-Holland, Amsterdam, 2002, pp. 423-461.

[RS14] Continuous selections of multivalued mappings, Recent progress in general topology. III, Atlantis Press, Paris, 2014, pp. 711-749. 
[Sch14] R. Schneider, Convex bodies: the Brunn-Minkowski theory, expanded ed., Encyclopedia of Mathematics and its Applications, vol. 151, Cambridge University Press, Cambridge, 2014.

Igor Belegradek, School of Mathematics, Georgia Institute of Technology, Atlanta, GA, USA 30332

E-mail address: ib@math.gatech.edu

$U R L$ : www.math.gatech.edu/ ib

Mohammad Ghomi, School of Mathematics, Georgia Institute of Technology, AtLANTA, GA , USA 30332

E-mail address: ghomi@math.gatech.edu

$U R L$ : www.math. gatech. edu/ ghomi 